\title{
MEASURING DEFORMATIONS IN THE TELESCOPIC BOOM UNDER STATIC AND DYNAMIC LOAD CONDITIONS
}

\author{
Cristiano Fragassa, Giangiacomo Minak, Ana Pavlovic
}

Department of Industrial Engineering, University of Bologna, Italy

\begin{abstract}
The interest in pushing the mechanical structures closer to their limits of usage makes necessary to combine the traditional design with the implementation of specific tests able to definitely confirm and guarantee their safety. Exploring the case of a large telescopic boom, the present study analyses the response to intense loads prevenient from static and dynamic conditions. The measure of deformations was oriented to validate several design assumptions, but also to investigate the presence of phenomena of local instability, not easily predictable within theoretical formulations.
\end{abstract}

Key Words: Telescopic Arm, Experimental Mechanics, Deformation Measurement, Metal Structures, Buckling, Mechanical Hysteresis

\section{INTRODUCTION}

The possibility of using mechanical structures in the load situations near the resistance limits of their materials represents, on one hand, a real opportunity for improving their functionality, and, on the other hand, a serious risk that only a perfect design can prevent. This is precisely the case of the telescopic boom object of this study.

Among the diverse applications for which a telescopic boom can be designed [1-3], this one is intended to be firstly mounted on fire trucks. In this case, the ability to work very close to the material limits, which involves perfect management of sheets thickness $[4,5]$, contacts and sliding forces, aiming at reducing the risks of instability [6-8], allows the design of ever-slimmer and lighter structures. Or, in different terms, keeping an identical weight, the same design requirements make it possible to create structures with ever-increasing outreaches, enlarging their functionality [2, 3].

Received December 01, 2018 / Accepted January 15, 2019

Corresponding author: Cristiano Fragassa

Department of Industrial Engineering, Alma Mater Studiorum University of Bologna, viale Risorgimento 2, 40136, Bologna, Italy

E-mail: cristiano.fragassa@unibo.it 
This general concept is not as simple as it may appear in terms of safety design $[9,10]$. Mechanical structures are often designed through a 'classic approach' which provides for various simplifications and ways such as the determination of the safety coefficient [1]. However, the same concepts are likely to be overcome when structures are designed for extreme conditions of use, since other unpredicted phenomena can occur [11, 12]. Referring, for instance, to the case of the thin metal sheets the telescopic boom is made of, the more their thickness is reduced, the more unstable their behavior becomes [13]. Thus, every action dealing with a reduction in thickness needs to be thoroughly analyzed by theories including the study of second order effects, such as buckling of plates and sections [14-17].

An alternative solution is represented by the massive use of numerical calculations by means of tools such as FEM algorithms [16-18]. However, also in this case the information available can be non-conclusive, leaving the execution of validation tests as a last resource. In this paper, a complex experiment is defined and detailed with the scope to validate the functionality and the level of safety offered by a large telescopic boom. External loads were applied and deformations measured in such a way as to investigate the overall behavior of the boom in static and dynamic conditions, together with the presence of unattended phenomena of instability.

\section{MATERIALS AND METHODS}

\subsection{The Case-study}

This investigation is based on a specific telescopic boom, installed on relatively light vehicles and used for moving aerial platforms on fire trucks (but can be used with other vehicles as well), and which provide:

- Working height: $17 \mathrm{~m}$

- Platform height: $15 \mathrm{~m}$

- Load capacity: $200 \mathrm{~kg}$

- Outreach: $9 \mathrm{~m}$

In particular, the research intends to join experimental evidence with the theoretical and FEM considerations already available from a previous study [19] with the aim to improve the overall comprehension of the structure response in respect to working loads. In this sense, it is specifically interesting to validate with measures the limits of utilization of the telescopic boom in terms of its structural resistance and stability.

The experiment started with the development of a structural reconstruction of the complex mechanism used for joining the different parts of the telescopic boom and consisting of two rectangular tubes coupled by two pairs of sliding pads (Fig. 1a). This connection was mounted inside special equipment specifically designed to provide conditions of forces and constrains representative of the real telescopic boom during its usage (Fig. 1b). In particular, the double hinge offers a situation of fix constrain on one side while the other side is pressed by two pistons in contraposition (Figs. 1c and 1d), moved by hydraulic pumps and able to provide a vertical force up to 200 bar each. The full equipment made by welding was $5 \mathrm{~mm}$ sheets of AISI4130 steel. 

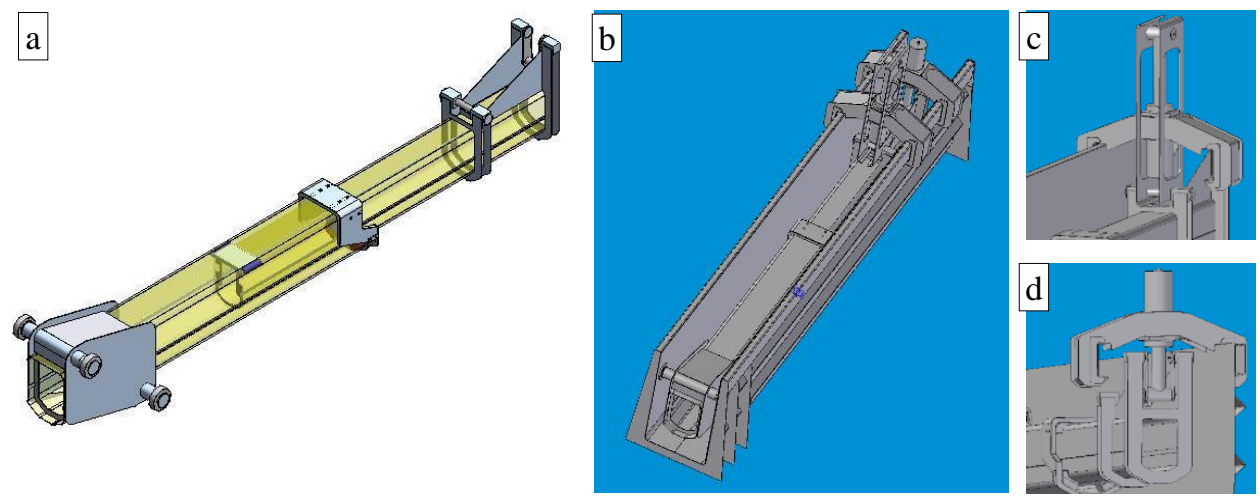

Fig. 1 Experiment design: (a) model of boom showing tubular parts, slides and supports; (b) boom inside the testing equipment; (c) fixing and (d) loading systems

The theoretical evaluation of forces and moments was carried out in [19] simplifying the model by the use of a beam, rigidly fixed in one side (Fig. 2a). This simplification deals with the evidence that the straight prismatic geometry is limited by two flat perpendicular bases, able to generate side surfaces very close to the points of application of loads. In addition, since mass forces are nearly irrelevant compared to the external loads and since different sections are free from constraints for the largest part of their length, the system can be led back to a Lamé 's Problem

Furthermore, in accordance with the De Saint Venant's Principle, in the presence of elastic materials (as in this case considering the properties of the steel), it is possible to replace a given system of forces with another one, having the same resultant force and the same resultant torque since diverse applications and distribution of these loads do not significantly influence the beam in the sections far from the point of application [19].

Then, in brief, this system can be considered as a slender beam, namely a solid having one predominant dimension comparing to the others, undergoing small displacements and with loads and constraints applied at the ends.

An additional stage in the simplification is permitted by the reduced thickness of the sheets that allow the adoption of the De Saint Venant's theory for the beam with thinwall. The related shear force and bending moment are displayed in Fig. 2b.

a

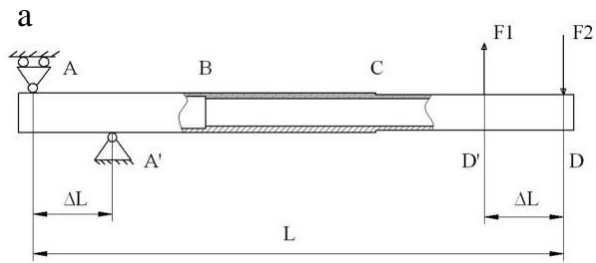

b

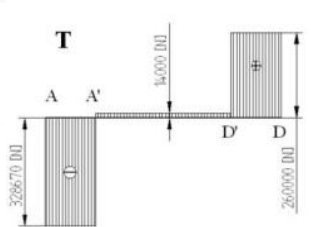

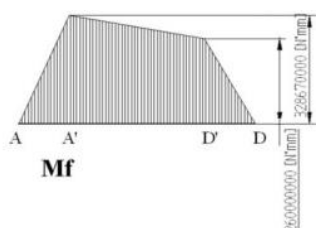

Fig. 2 Design of the experiment: (a) scheme of forces and constrains and (b) diagram of shear force $(T)$ and bending moment $(M f)$ 

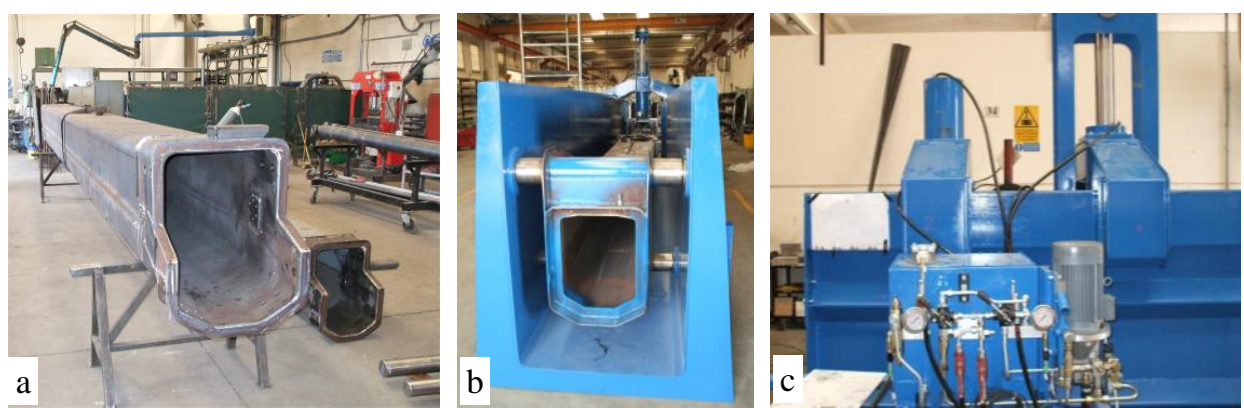

Fig. 3 Images of the telescopic boom: (a) the two extensions of the boom in the workshop while waiting to be assembled; (b) the machine during the tests with the supporting frame in the foreground; and (c) the hydraulic system for the application of external forces

\subsection{Acquisition of deformations}

In the experiment, 15 strain gauges were used with the scope to investigate the response of the structure to loads. Thanks to a variation of electrical resistance of the monoaxial filament, these strain gauges are able to transform the deformations of the structures they are glued over into electric signals. These strain gauges have innumerable advantages including the fact that they can be placed on the surfaces with any spatial orientation and, if protected by silicone, can remain long even in humid and dusty environments, and thus can be used for more tests. They are also simple, economical and precise sensors that lend themselves particularly well to the measurement of static and possibly dynamic deformations on the surface of the loaded bodies.

\subsection{Placement of strain gauges}

Taking into account the results of the previous simulations [19], the strain gauges were placed in strategic points, particularly suitable for monitoring the behavior of the telescopic boom subject to bending load (Fig. 4).

Entering in details, strain gauges 1, 2, 3 and 4, considered important for analyzing possible areas of stress concentration, were glued to the lower part of the inner arm, in correspondence with the sliding pads. Strain gauges 1 and 3 longitudinally and strain gauges 2 and 4 were placed transversely on the two sides of the beam, in non-horizontal areas near the edges. Strain gauge 15 was placed on the opposite side with respect to the previous ones, on the same section with control functions. Strain gauges 5 and 6 were glued symmetrically on the upper and lower part of the inner beam, respectively. The same was done with strain gauges 11 and 12 for the external beam. In these areas, there was no provision for stress concentrations. Strain gauges 7 and 8 were located on the outer beam at the sliding pads as well as strain gauges 9 and 10 otherwise left in reserve. Strain gauges 13 and 14 were glued on the side in an area which is supposed to present problems of instability of the elastic equilibrium. After cleaning and smoothing the surfaces, the strain gauges were glued with acrylic glue and then covered with silicone in order to remain protected during the assembly phase; in this way it became possible to use them for any subsequent experimental campaigns. Some of the internal strain gauges were fitted before 
the boom was assembled, paying attention that the high temperatures reached during welding would not compromise their functionality.

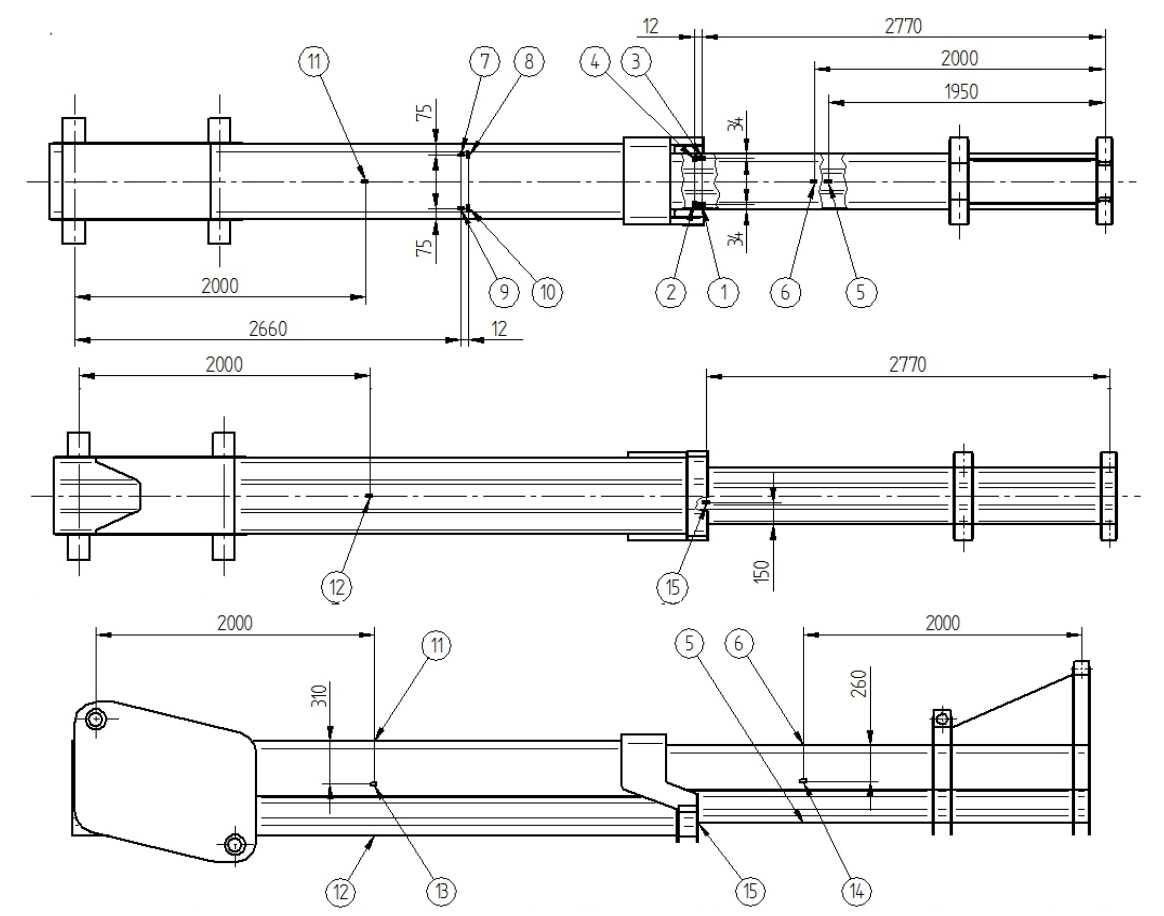

Fig. 4 Position and orientation of the strain gauges

\subsection{Anticipating the measurements}

Bearing in mind the position of the strain gauges, with simple considerations derived from the lean beam theory and symmetry conditions it was expected that the:

- strain gauges 1 and 3 detect similar maximum values, as well as 2 and 4;

- strain gauges 5 and 6 show similar maximum values, but with opposite sign;

- strain gauges 11 and 12 offer the same situation of strain gauges 5 and 6 ;

- strain gauges 13 and 14 show very low values compared to the others;

- strain gauge 15 detects a value quite similar to that of strain gauges 1 and 3 .

\subsection{Calibration of the experimental system}

A preliminary operation was carried out to check for any variations in the 'zero' values read by the internal strain gauges, which were placed before the telescopic arm was assembled. In this way, it was verified that the residual stresses due to the welding of the sheets and the deformations related to the assembly did not significantly interfere with the results of the measurements. In particular, a doubt was related to the possibility that the high temperatures reached during welding could damage the internal strain gauges ( $1,2,3,4$ and 5), despite the accurate cooling system developed to prevent this 
problem. However, a test carried out with an infrared sensor ensured that the sheets on which the strain gauges were applied did not exceed $50{ }^{\circ} \mathrm{C}$, a fairly low value compared to a maximum permissible temperature of about $90{ }^{\circ} \mathrm{C}$.

As the next step, the acquisition system was calibrated starting with the calibration of the pressure sensors that were checked by respective instrument. It was also verified that their response remained proportional to the pressure rise. Then, the calibration moved to the strain gauge system, recording the values read in the absence of external load, but with the arm subjected to its own weight. A first load cycle at 10 bar with the discharge after the nominal value of 0 bar was used to make the system settle and with the acquired values the strain gauges were zeroed.

\subsection{Application of loads}

Tests were carried out by loading the structure, a force hold and a subsequent unloading. In particular, the load sequences, represented in Fig. 5, consisted of:

- slow and progressive loading up to the $75 \%$ of nominal load in accordance with design conditions, equivalent to 75 bar in the pumps pressure, including different stabilization and measuring points;

- progressive loading up to nominal design conditions;

- fast loading and unloading cycles up to the nominal load;

- loading and unloading cycles with maximum load increase, 200 bar, equal to 3 times the nominal load;

- the zeroing of the strain gauges was finally carried out and two tests were carried out, increasing the imbalance between the pumps at 61-39 bar and 106-85 bar respectively.

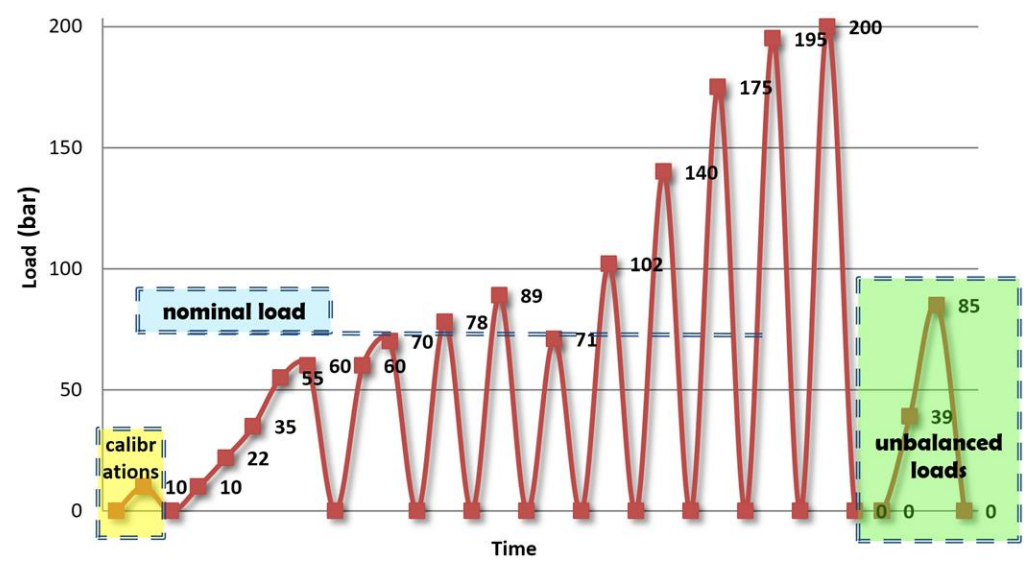

Fig. 5 Loading and unloading cycles carried out during the tests 


\section{RESULTS}

\subsection{Deformations at nominal load}

Figure 6 shows the typical result of an acquisition (in the case of nominal load of 75 bar). First of all, it can be observed that all strain gauges work regularly with no open circuit effects or perceptible noise on the signals, further confirming that the welding and assembly operations did not cause damage to the sensors. Strain gauges 9 and 10 were not acquired, but kept in reserve.

The pressure trends in the two pumps can also be noted (in black and with values obtained on the right side): it is clear how, before reaching the nominal pressures, oscillations due to the inertia of the fully manual regulation hydraulic system appear.

By enlarging the loading phase (Fig. 6b), it is possible to see that the load reaches its maximum in a not completely regular manner and the pumps remain, even if slightly, unbalanced. To improve clarity, only a reduced set of strain gauges is visible.

Observing the measurements, it immediately becomes evident that there are two "classes" of strain gauges that behave differently. Strain gauges 1, 3, 5, 6, 15 (axial) follow almost perfectly the load trend, apart from a slight hysteresis linked to the hydraulic circuit and to the gaps present in the arm; in particular, strain gauge 6, which is far from the covering area, also follows the smallest oscillations of the load (Fig. 6b, top). On the contrary, strain gauges 2, 4, 7, 8 (transverse) show a marked hysteresis phenomenon, so that the deformation peaks are delayed and the deformation continues to increase for a while even when the load decreases (Fig. 6b, right).

It is very likely that this phenomenon is linked to a viscous effect caused by the presence of the sliding pads, which delay the transfer of load between the two extensions of the arm and, in the case of a strong peak, they considerably attenuate it.
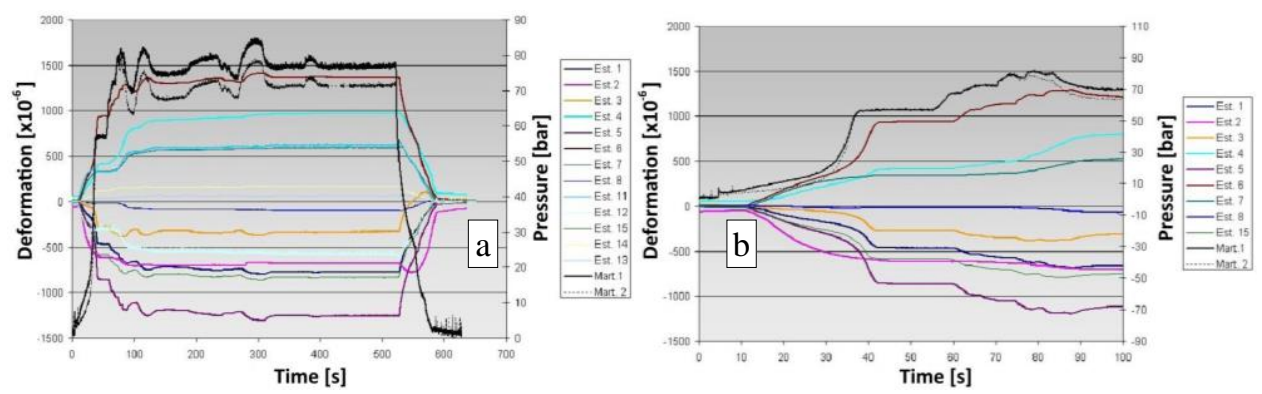

Fig. 6 Measures for nominal load (75 bar): (a) entire spectrum and (b) loading phase only

Comparing measures with expectations:

- strain gauges 1-3 and 2-4 have different readings showing that the part of the expected symmetry is missing in the real structure or loads (or both);

- strain gauges 5 and 6 actually detect similar values with opposite sign, as well as 11 and 12: De Saint Venant's theory is applicable in those areas;

- strain gauge 6, as expected, detects the maximum deformation;

- strain gauges 13 and 14 correctly detect lower values (-13\%) than strain gauge 6;

- strain gauge 15 has a measure similar to strain gauge 1, but not strain gauge 3 . 
It is possible to conclude that, for the nominal load conditions:

1. the stress state is similar to that of a slender beam only far from the covering area;

2. there is no evidence of instability phenomena;

3. the loading of the structure appears not symmetrical (or, even if less probable, the structure has internal constraints such as to make its geometry not symmetrical): this asymmetry generates an undesired twisting moment on the arm with longitudinal axis which must be taken into account;

4. a deformation concentration effect on the sliding pads appears, which can be evaluated as the average of measures from strain gauges 1-3 and 2-4.

\subsection{Measures at maximum load}

During the phase of loading up to the maximum load of 200 bar, already exceeding 140 bar noise emissions were noticed both in the loading and unloading phases. In correspondence with these noises, the strain gauges positioned at the sliding pads (in particular the 2 and 4 ) recorded jumps (as can be seen in the red circles in Fig. 7).
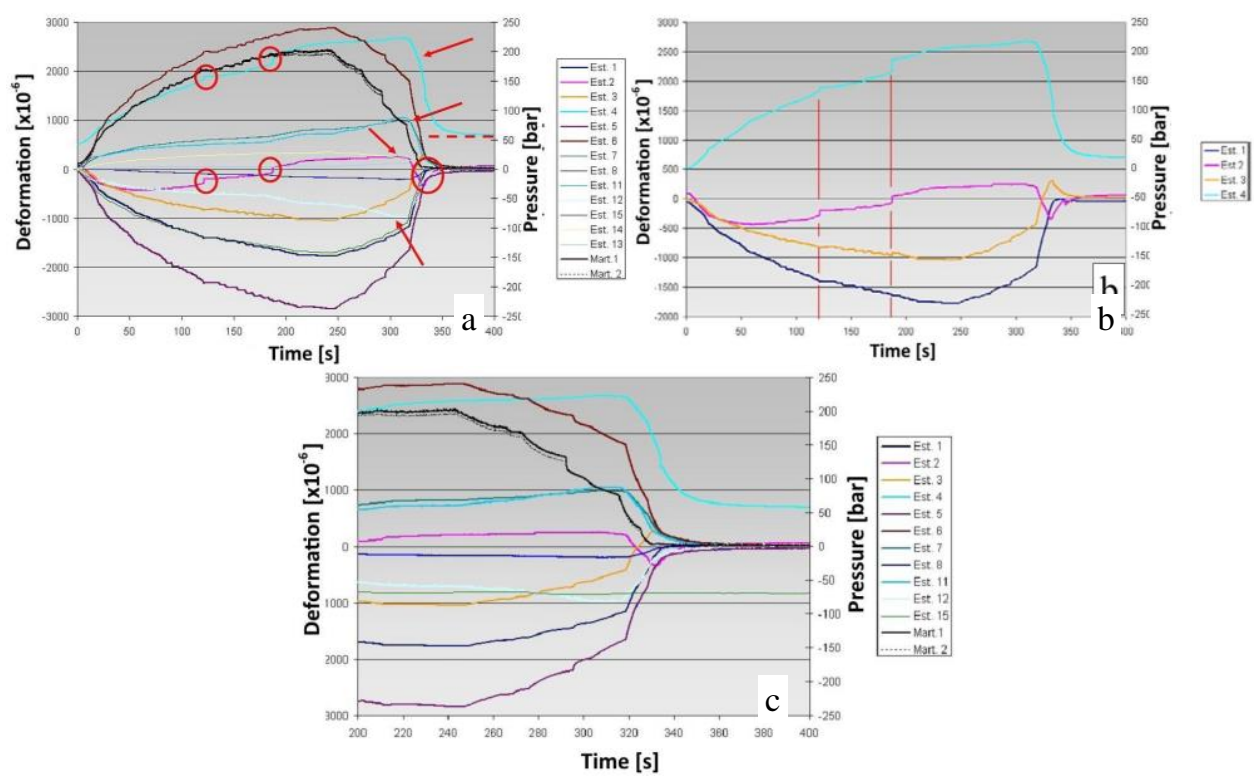

Fig. 7 Measures for maximum load (200 bar): (a) whole spectrum with stick-slip phenomena highlighted; (b) simultaneous jumps on all strain gauges; (c) unloading phase

The most plausible explanation for these events is related to the presence of a stick-slip phenomenon (sliding) between the pads and the internal arm, which could most likely be eliminated by means of suitable lubrication. Due to the friction of the first detachment, elastic energy is stored which is released and suddenly deforms the arm emitting an acoustic wave at the same time, following a small slipping. Since they were static tests, putting sliding pads was not taken into consideration. However, the measurements were not compromised by this effect since the energy accumulated had extremely low values and was immediately released.

In Fig. $7 \mathrm{~b}$ it is possible to better appreciate this behavior in strain gauges 1,3 (very light) and 2, 4 (accentuated) where it can be noted that all the strain gauges reveal the phenomenon 
at the same time through vertical lines. Instead, in Fig. 7c the trend of the readings in the unloading phase of the structure can be observed twice. While the strain gauges positioned in the areas far from the pads faithfully follow the load, those at the covering have a marked hysteresis to be attributed to the viscoelastic behavior of the ertalon and to the stick-slip phenomena. It should be noted, for example, that while strain gauge 6 closely follows the discharge, strain gauge 4 remains charged for a certain period, even if the arm is being unloaded, because the pad is opposed by friction upon returning to the condition of rest. Suddenly, the deformation drops with an intermediate step in correspondence of which the acoustic emission is also recorded, and residual deformation remains, which, however, is only slightly higher than that present at the beginning of the loading phase. But it is not only strain gauge 4 that has such a particular behavior: it is seen that strain gauges 2 and 3 even change their sign before returning correctly to zero, highlighting effects of rebound of the structure.

From the quantitative point of view, it is observed that the maximum deformation recorded is about $2.9 \cdot 10^{-3}$ (strain gauge 6), corresponding to $600 \mathrm{MPa}$ in terms of stress, way below the yield stress. Finally, strain gauges 13 and 14 , which had to control the onset of elastic equilibrium instability, present no deviation from the linearity, remaining at strain values lower than $0.35 \cdot 10^{-3}$, also experimentally denying the risk of structural buckling.

\subsection{Measures for unbalanced loads}

During the simulations, it was noticed how slight unbalancing loads between pumps 1 and 2 significantly overload the boom with relevant shear stresses. Fig. 8 shows the results of a test with progressively rising loads with an imbalance of $25 \%$, until reaching, on the two pumps, 106 and 85 bar, respectively.
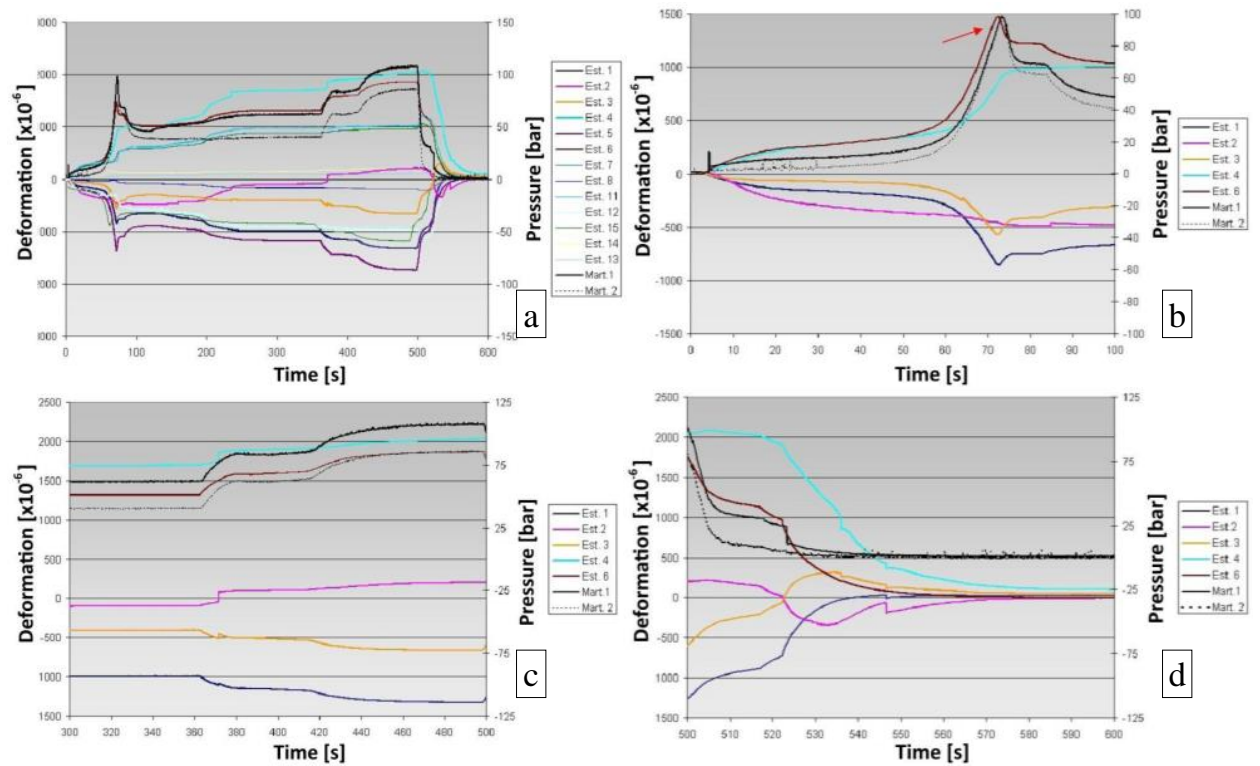

Fig. 8 Measures for "unbalanced" loads: (a) entire spectrum; (b) correspondence between load trends and deformations; (c) loading and (d) unloading phases 
In particular, in Fig. 8b shows different responses of the strain gauges to the initial pressure peak, not detected by strain gauges 2 and 4 , as if these sensors mediated what happened to the structure by charging and discharging more slowly, but detected by 1 and 3 (even if in a slightly attenuated form). In Fig. 8c it is possible to see the stick-slip phenomenon again: in fact, in the face of a load increase, while strain gauge 6 follows the ramp perfectly and strain-gauges from 1 to 4 are little affected, they have a discontinuity in the deformation. The usual behavior, with steps and inversions of sign, is visible in the unloading (Fig. 8d) where, following re-adjustments in the geometric configuration of the arm, continue strong sound emissions that persist for a few seconds even at nominal load virtually nil are present.

\subsection{Load - deformation curve}

The load-deformation curve shows the amplitude of the structure's hysteresis and its tendency to accumulate energy. The reported case is the one recorded at 90 bar by the two strain gauges 11 (black) and 12 (red) (Fig. 9). It is possible to observe the first phase of linear loading followed by a phase in which, when the pressure increases (between 20 and 90 bar), the deformation remains constant. In this phase, the recovery of the mechanical and 'hydraulic' gap and the crushing of the sliding pads are present. Then, the level of deformation starts to increase.

The discharge phase is more regular except for an initial increase in the deformation due to the unbalancing of the pumps during unloading. With the current loading system, which controls only the pressure in the cylinders, it is not possible to carry out direct loaddeformation graphs from which to draw conclusions on local effects because there is a large hysteresis area so as not to allow the perception of the real trend of the forces, and therefore of the bending moment.

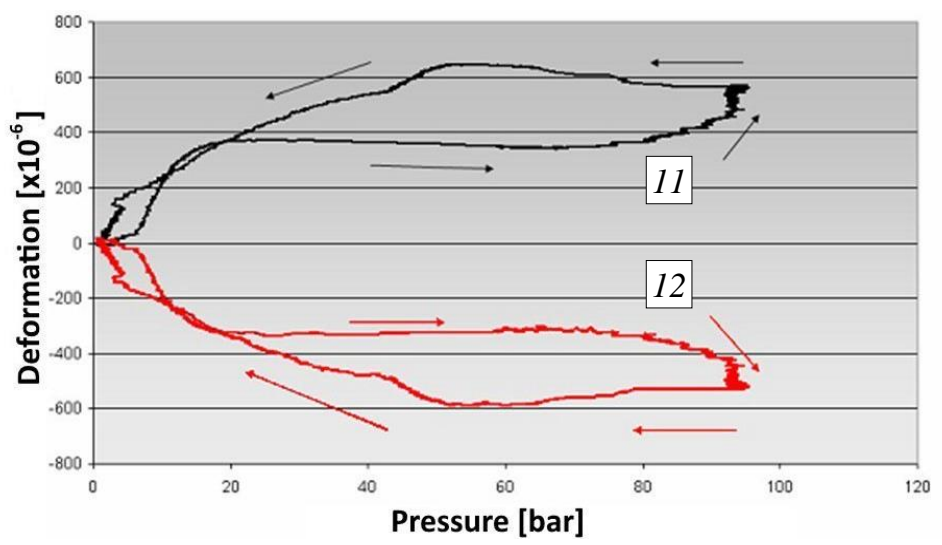

Fig. 9 Load-deformation curve with evidence of hysteresis (only two strain gauges) 


\section{DISCUSSION}

\subsection{Global behavior}

Every time that a stable pressure condition was reached, with a measuring tape able to guarantee an accuracy of the millimeter, the distance between the bottom of the box and the loaded end of the beam was measured obtaining the total inflection of the arm according to the applied pressure (Fig. 10a). This curve was compared with the measures from strain gauges of maximum deformations as a function of the bending moment applied (Fig. 10bd). Both show non-linear initial behavior of the system with large displacements for small load values followed by a fairly linear tendency. The initial trend is certainly linked to the recovery of gaps, the torsion caused by the asymmetry of the system and the viscoelastic behavior of the sliding pads in ertalon. As the load increases, the behavior of the beam as a whole changes and, in the case of strain gauges near the sliding pads, due to the lack of symmetry of the actual contact conditions, the deformation is also qualitatively different between the symmetrically applied strain gauges. In Fig. 10b, for instance, it is evident that strain gauge 2, placed transversely in correspondence with one of the lower sliding pads, diverges from linearity with even changing its sign, while strain gauge 4 has values much higher than its symmetrical.
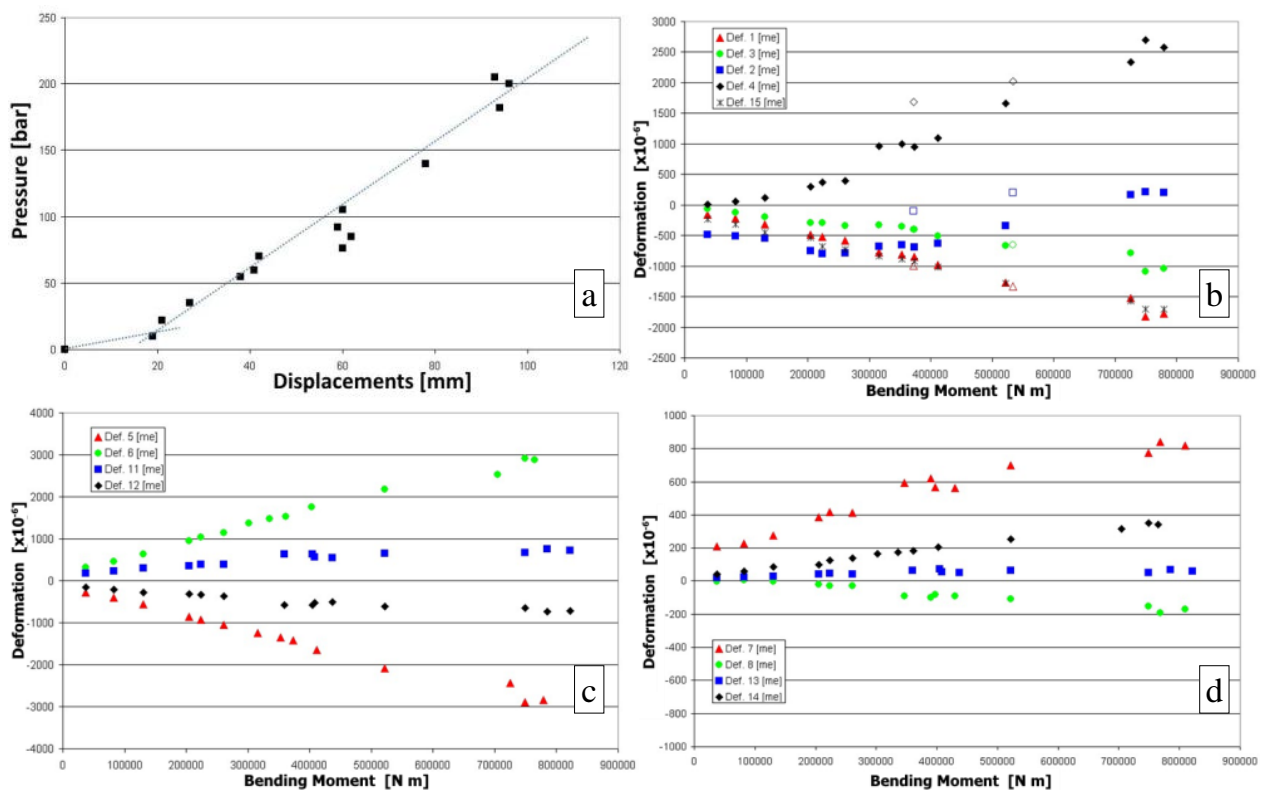

Fig. 10 Parallelism between the (a) overall behavior and (b-d) local deformations

By looking at the trends of strain gauges 2 and 4, the hypothesis that the arm is subjected to torque becomes more and more solid, considering how the first tends to discharge while the second is overloaded.

Even visually, at the maximum loads applied (over 140 bar), the effects of torsion on the loaded end of the arm began to be noticed (Fig. 11). 


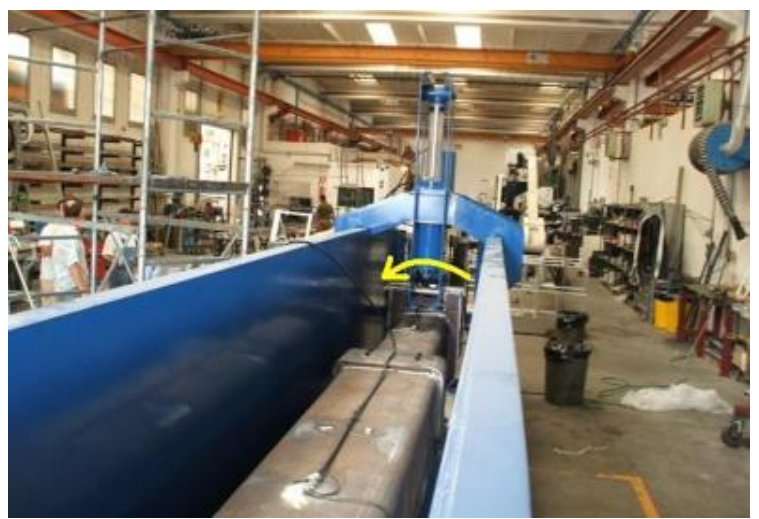

Fig. 11 Telescopic arm under test with the first effects of torsion

\subsection{In brief}

As synthesis of experimental measures, it is possible to say that:

1. all strain gauges worked correctly,

2. the steel structure remained in the linear field without presenting instability of the elastic balance even for loads equal to almost 3 times the nominal one,

3. the presence of plastic sliding pads and mechanical gaps has determined a reduction of the overall rigidity and the non-linearity in the first stretch (with viscous sliding phenomena) of the load-displacement curve,

4. most of the measures are compatible with the beam theory (without prejudice to the errors on the measurement of the load) except those of the strain gauges placed close to the sliding pads,

5. the apparent anomalous measures of strain gauges 2 and 4, placed near the sliding pads, are linked to local effects such as load redistribution, slippages and viscous effects due to pads, which create an asymmetry in the local situation. Combining the measures from strain gauges 2 and 4 , these effects can be almost compensated,

6. The detection of the loads applied to the arm through custom-made load cells, instrumenting both the connecting rods and the supports of the shoes, would allow to circumvent the hysteresis problems of the hydraulic circuit.

\subsection{Theory vs. Experiment}

There are both correspondences and differences between forecasts from theory or simulation and measurements through which it is possible to move toward a better understanding of what happens inside a telescopic boom, especially in the connecting areas. In particular, from this comparison, it is possible to clarify that:

a. in the areas away from contacts (representing the largest part of the metal sheets), the variations between measures and theory are minimal, practically confirming the presence of a membrane state of stress,

b. strain gauge 15 shows a tension much higher than expected which shows a strong transversal deformation in the area between the lower pad, able to influence the reading of the strain gauge (which otherwise would have had to provide only the axial strain), 
c. the asymmetry of the applied loads, most likely mainly due to geometric imprecisions of the supports and during the assembly, generated a torque which did not allow in some points a direct comparison between the results of the numerical simulations and the direct measure of the directional deformations, but only between their average values,

d. by appropriately correcting the simulation model to take into account the real asymmetry that presents the contact area (explained above), the numerical method can lead to extremely accurate results (e.g. close to strain gauges 1 and 3, a -116 $\mathrm{MPa}$ was measured, very close to the $-120 \mathrm{MPa}$ of the simulation, as well as around 2 and 4 where $31 \mathrm{MPa}$ are detected against the 38 simulated $\mathrm{MPa}$ ),

e. with a peak of $728 \mathrm{MPa}$ estimated in the contact area under nominal load conditions, the telescopic boom is always in the elastic range (stresses lower than $1300 \mathrm{MPa}$ ) and can operate without particular problems,

f. as the load increases, the soft sliding pads in ertalon soon reach (as expected) the yield stress and, deforming, enlarge the contact area and redistribute the stress in the steel by lowering the localized peaks: in the upper surface of the internal extension (the most critical for the strength of the structure) not even reaching $300 \mathrm{Mpa}$,

g. with maximum load of almost 3 times the nominal one, the strains on the external extension provide axial tension values that are about $140 \%$ less than those obtained with the simulation. The most plausible hypothesis leads to consider how on the external extension, which has a perfectly fitted end with two sleeves, the (unwanted) torque is most perceived by its sensors. They only record the longitudinal component of a deformation that instead has an inclination of $45^{\circ}$ with respect to the axis. The internal extension is less affected by this effect due to the lower stiffness of the shoes,

h. it is believed that by improving loads on sliding pads, the plasticized part would have affected the fittings with the lower surface and with the side walls extending the section involved in the covering and reducing the stresses,

i. there are no instability phenomena up to the maximum load of 200 bar and, in general, it is believed that geometric instability cannot occur because plasticization problems would arise before,

j. in accordance with the simulations where a pure bending moment was provided, the boom should collapse under a pure moment above $1000 \mathrm{kN} \cdot \mathrm{m}$, corresponding to 300 bar (or for a moment combination of $923 \mathrm{kN} \cdot \mathrm{m}$ and share force of 52,570 kN). These load values, 4 times higher than the design limits and far from the potentialities of the present testing equipment, cannot reasonably be achieved during operation conditions by the structure, even assuming abrupt or unconventional overloads.

\section{CONCLUSIONS}

The theoretical and FEM modeling studies, together with the noticeable experience of the designers, allowed to devise an important and complex experiment that, involving over $7000 \mathrm{~kg}$ of sheets, aimed to understand the mechanical behavior of the telescopic arms used in the aerial platforms. Combining the results from theory and simulations with these measures, it was possible to refine the interpretative model creating new tools able to support the design of safer and more efficient structures. In conclusion, the experimental 
tests have validated the numerical simulations, which therefore can be used reliably in the design and verification of structures similar to the one examined.

Acknowledgements: The authors wish to thank Alessandro Fantuzzi and Marco Faedi for their essential contribution in the experiment design and implementation, and Vanda Roversi and Dino Balduzzi for their support in signal measuring and elaboration.

\section{REFERENCES}

1. EN 280:2000 standard, Mobile elevating work platforms. Design calculations. Stability criteria. Construction. Safety, Examinations and tests.

2. Włodzimierz, S., 2003, Mobile platforms, Construction and exploitation, Krosno KaBe

3. Joyce, N. 1995, Design of a mobile elevating work platform, M. Phil Thesis, Brunel University.

4. Timoshenko, S. P., Gere, J. M., 2009, Theory of elastic stability, Courier Corporation.

5. Tvergaad, T., 1999, Studies of Elastic-Plastic Instability, Journal of Applied Mechanics, 66, pp. 3-9.

6. Mitrev, R., Janošević, D., Marinković, D., 2017, Dynamical modelling of hydraulic excavator considered as a multibody system, Tehnički vjesnik, 24(2), pp. 327-338.

7. Bažant, Z, Cedolin, L, 2010, Stability of structures: elastic, inelastic, fracture and damage theories, World Scientific.

8. Arbocz, J., Weller, T., 1998, Buckling Experiments, Basic Concepts, Columns, Beams and Plates, John Wiley\&Sons.

9. Abraham, J, Sivaloganathan, S., Rees, D.W.A., 2011, The Telescopic Cantilever Beam: Part 1 Deflection Analysis, Engineering Integrity, 30, pp. 6-15

10. Abraham, J, Sivaloganathan, S., Rees, D.W.A., 2011, The Telescopic Cantilever Beam, Part 2 - Stress Analysis, Engineering Integrity, 31, pp. 6-17.

11. Derlukiewicz, D., Karliński, J., 2012, Static and dynamic analysis of telescopic boom of self-propelled tunnelling machine. Journal of Theoretical and Applied Mechanics, 50(1), pp. 47-59.

12. Huang, X.L., Ji, A.M., 2013, Analysis of Nonlinear Local Buckling of Crane Telescopic Boom, Applied Mechanics and Materials, 387, pp. 197-201.

13. Miao, Q., Zhang, Z.P., Xie, F., Li, X., 2013, Boom Buckling Instability Capability Studies. Applied Mechanics and Materials, 385, pp. 316-319.

14. Yao, J., Qiu, X., Zhou, Z., Fu, Y., Xing, F., Zhao, E., 2015, Buckling failure analysis of all-terrain crane telescopic boom section, Engineering Failure Analysis, 57, 105-117.

15. Jeevan, G.A., 2012 A Deflection, Buckling and Stress Investigation into the Telescopic Cantilever Beam, $\mathrm{PhD}$ thesis, School of Engineering and Design, Brunel University.

16. Derlukiewicz, D., Przybyłek, G.., 2008, Chosen aspects of FEM strength analysis of telescopic jib mounted on mobile platform, Automation in construction, 17(3), pp. 278-283.

17. Janošević, D., Pavlović, J., Jovanović, V., Petrović, G., 2018, A numerical and experimental analysis of the dynamic stability of hydraulic excavators, Facta Universitatis-Series Mechanical Engineering, 16(2), pp. $157-170$

18. Aimin, J., Peiqiang, Z., Duo, P., Yanling, L., 2004, Finite Element Analysis for Local Stability of Telescopic Boom of Truck Crane, Transactions of the Chinese Society of Agricultural Machinery, 35(6), pp. 48-51.

19. Pavlovic, A., Fragassa, C., Minak, G., 2017, Buckling Analysis of Telescopic Boom: Theoretical and Numerical Verification of Sliding Pads, Tehnicki Vjesnik, 24(3), pp. 729-735. 